\title{
High gene expression levels of VEGFA and CXCL8 in the peritumoral brain zone are associated with the recurrence of glioblastoma: A bioinformatics analysis
}

\author{
XIAOBIN LUO ${ }^{1}$, SHANGYI XU ${ }^{1}$, YALI ZHONG ${ }^{2}$, TIANQI TU ${ }^{1}$, YOULIN XU ${ }^{1}$, \\ XIANGLONG LI ${ }^{1}$, BIN WANG ${ }^{1}$ and FUBING YANG ${ }^{1}$ \\ ${ }^{1}$ Department of Neurosurgery, The Affiliated Hospital of Southwest Medical University, Luzhou, Sichuan 646000; \\ ${ }^{2}$ School of Nursing, Guizhou University of Traditional Chinese Medicine, Guiyang, Guizhou 550000, P.R. China
}

Received April 25, 2019; Accepted September 17, 2019

DOI: 10.3892/ol.2019.10988

\begin{abstract}
The present study aimed to identify differentially regulated genes between the peritumoral brain zone (PBZ) and tumor core (TC) of glioblastoma (GBM), to elucidate the underlying molecular mechanisms and provide a target for the treatment of tumors. The GSE13276 and GSE116520 datasets were downloaded from the Gene Expression Omnibus (GEO) database. Differentially expressed genes (DEGs) for the PBZ and TC were obtained using the GEO2R tool. The bioinformatics and evolutionary genomics online tool Venn was used to identify common DEGs between the two datasets. The Database for Annotation, Visualization, and Integrated Discovery online tool was used to analyze enriched pathways
\end{abstract}

Correspondence to: Professor Fubing Yang, Department of Neurosurgery, The Affiliated Hospital of Southwest Medical University, 25 Taiping Street, Jiangyang, Luzhou, Sichuan 646000, P.R. China

E-mail: fubingyang1@hotmail.com

Abbreviations: GBM, glioblastoma; PBZ, peritumoral brain zone; TC, tumor core; rGBM, recurrent glioblastoma; GEO, Gene Expression Omnibus; DEGs, differentially expressed genes; GO, Gene Ontology; KEGG, Kyoto Encyclopedia of Genes and Genomes; PPI, protein-protein interaction; UP, upregulated; DOWN, downregulated; GEPIA, Gene Expression Profiling Interactive Analysis; TCGA, The Cancer Genome Atlas; GTEx, Genotype-Tissue Expression; HR, hazard ratio; PLP1, proteolipid protein 1; MOBP, myelin-associated oligodendrocyte basic protein; CNTN2, contactin 2; CXCL8, C-X-C motif chemokine ligand 8; MOG, myelin oligodendrocyte glycoprotein; VEGFA, vascular endothelial growth factor A; MBP, myelin basic protein; MAG, myelin associated glycoprotein; SOX10, SRY-box 10; PLLP, plasmolipin; IL-8, interleukin 8; MMP2, matrix metalloproteinase 2; CXCR, C-X-C chemokine receptor; B1R, B1 bradykinin receptor; TMZ, temozolomide

Key words: peritumoral brain zone, tumor core, bioinformatics analysis, recurrent glioblastoma, vascular endothelial growth factor A, C-X-C motif chemokine ligand 8 of the Gene Ontology (GO) and Kyoto Encyclopedia of Genes and Genomes (KEGG) databases. The Search Tool for the Retrieval of Interacting Genes/Proteins online tool was used to construct a protein-protein interaction (PPI) network of DEGs. Hub genes were identified using Cytohubba, a plug-in for Cytoscape. The Gene Expression Profiling Interactive Analysis (GEPIA) database was utilized to perform survival analysis. In total, 75 DEGs, including 12 upregulated and 63 downregulated genes, were identified. In the GO term analysis, these DEGs were mainly enriched in 'regulation of angiogenesis' and 'central nervous system development'. Furthermore, in the KEGG pathway analysis, the DEGs were mainly enriched in 'bladder cancer' and 'endocytosis'. When filtering the results of the PPI network analysis using Cytohubba, a total of 10 hub genes, including proteolipid protein 1, myelin associated oligodendrocyte basic protein, contactin 2, myelin oligodendrocyte glycoprotein, myelin basic protein, myelin associated glycoprotein, SRY-box transcription factor 10, C-X-C motif chemokine ligand 8 (CXCL8), vascular endothelial growth factor A (VEGFA) and plasmolipin, were identified. These hub genes were further subjected to GO term and KEGG pathway analysis, and were revealed to be enriched in 'central nervous system development', 'bladder cancer' and 'rheumatoid arthritis'. These hub genes were used to perform survival analysis using the GEPIA database, and it was determined that VEGFA and CXCL8 were significantly associated with a reduction in the overall survival of patients with GBM. In conclusion, the results suggest that the recurrence of GBM is associated with high gene expression levels VEGFA and CXCL8, and the development of the central nervous system.

\section{Introduction}

Originating from adult brain tissue, glioblastoma (GBM) is a highly malignant primary tumor with numerous characteristics, including complicated pathological diagnosis, poor prognosis and easy relapse (1). Tumor cells have a strong ability for proliferation and invasion, and can invade the central nervous system (2). In 2018, patients with GBM had a poor prognosis, and the median survival time of patients was $\sim 17$ months (3). In 2019, following standard treatment, the 
6-month progression-free survival rate was $25.2 \%$ in patients with recurrent glioblastoma (rGBM) (4). Traditional treatment for GBM includes surgical resection, radiation and chemotherapy with temozolomide (5). Following surgical resection of GBM, substantial invading tumor cells are located in the near resection edge of the tumor or within $2-3 \mathrm{~cm}$ from the resection cavity, and these are the most common recurrence sites (6).

In previous years, with the development of medicine and bioinformatics, gene expression information has been generated and stored in the Gene Expression Omnibus (GEO) database. Bioinformatics analysis allows an improved understanding of the alterations in essential genes in the process of tumor development. Furthermore, it can provide a basis for target treatment and tumor diagnosis (7). A previous study identified that both the peritumoral brain zone (PBZ) and tumor core (TC) are heterogeneous in the same patients, and tumor cells in the PBZ exhibit increased invasiveness compared with cells in the TC (8). Van Meter et al (9) demonstrated that there were a number of different gene expression products between the PBZ and TC. Even in the absence of tumor cells in the PBZ, it is possible to detect the expression of genes associated with invasion and neo-angiogenesis (10). Therefore, identifying differentially expressed genes (DEGs) between the PBZ and TC may reveal the causes of GBM recurrence, and may provide a target for tumor treatment.

In the present study, the gene expression profiles of the PBZ were compared with the gene expression profiles of the TC to identify DEGs. The DEGs of the PBZ and TC were screened by GEO2R online analysis, and the Database for Annotation, Visualization, and Integrated Discovery (DAVID) was used for Gene Ontology (GO) and Kyoto Encyclopedia of Genes and Genomes (KEGG) enrichment analyses. A protein-protein interaction (PPI) network was constructed using Search Tool for the Retrieval of Interacting Genes/Proteins (STRING) and Cytoscape software tools, and the hub genes were obtained using the Cytoscape plug-in Cytohubba. Furthermore, the Gene Expression Profiling Interactive Analysis (GEPIA) database was used to analyze the survival rate of patients with aberrant expression levels of the hub genes.

\section{Materials and methods}

Microarray data analysis. GEO (https://www.ncbi.nlm.nih. gov/geo/) is a database that stores a large amount of genetic information. For analysis, 'glioblastoma' was selected as a key word, the tissue type was set as 'Homo sapiens' and the study type was set as 'expression profiling by array'. The screening criteria were: i) Samples of peritumoral brain area and core of GBM; and ii) both samples should be obtained from the same patient. The two datasets that met the criteria were GSE13276 and GSE116520. The GSE13276 dataset, which was submitted by Mangiola et al (2), is based on the GPL96 platform and contains 5 cases of GBM core samples and 5 cases of GBM surrounding tissue (no replicate samples included). The GSE116520 dataset, which was submitted by Kruthika et al (11), is based on the GPL10558 platform and contains 17 cases of GBM core samples and 17 cases of GBM surrounding tissue (Table I).
Screening of DEGs. DEGs were screened using GEO2R (https://www.ncbi.nlm.nih.gov/geo/geo2r/), which allowed a comparison of the differences between two different sets of samples in a series. In the GSE13276 dataset, llog fold change (FC) $\mid \geq 2$ and $\mathrm{P}<0.05$ were defined as statistically significant for the DEGs. Similarly, in the GSE116520 dataset, $\log \mathrm{FCl}$ $\geq 1$ (and $\mathrm{P}<0.05$ were defined as statistically significant for the DEGs). If the same criteria in the two datasets were selected, the resulting genes would be limited, therefore in order to obtain a larger number of target genes different criteria were selected. Excel software (Office 2016; Microsoft Corporation) was used to delete all genes without gene symbols and duplicate genes corresponding to multiple probe IDs in the GSE13276 and GSE116520 datasets. A Venn diagram of the DEGs obtained from the bioinformatics and evolutionary genomics online tool 'Calculate and draw custom Venn diagrams' (http://bioinformatics.psb.ugent.be/webtools/Venn/) was constructed, and the common DEGs were identified.

GO and KEGG enrichment analysis. GO (http://www. geneontology.org) is a database used to describe the characteristics of genes and gene products. KEGG (https://www. kegg.jp/) is a database containing information on genome, chemistry and system functions (12). DAVID (version 6.8; http://david.ncifcrf.gov/) is an online database, and was used to perform GO term and KEGG pathway enrichment analyses $(13,14) . \mathrm{P}<0.05$ was considered to indicate a statistically significant difference.

Interaction network analysis and hub gene identification. The STRING (https://string-db.org/) database is a tool for analyzing the interrelationships between genes, including direct (physical) and indirect (functional) links, and constructing PPI networks. STRING version 11.0 contains $24,584,628$ proteins from 5,090 organisms (15). The PPI network association among DEGs was analyzed using the STRING database and the default minimum required interaction score was set as $>0.4$. Subsequently, the Cytoscape (version 3.7.1; http://www. cytoscape.org/) plug-in Cytohubba (version was 0.1; http://apps. cytoscape.org/apps/cytohubba) was used to screen the PPI network, and the top10 genes, which were the hub genes in the PBZ, were obtained using the Degree algorithm.

Survival analysis. GEPIA (http://gepia.cancer-pku.cn/detail. php) is a database used to analyze RNA sequencing expression data, and data of the tumors (GBM) and normal samples included in The Cancer Genome Atlas (TCGA: https://portal. gdc.cancer.gov/) and the Genotype-Tissue Expression (GTEx: https://gtexportal.org/home/) databases were analyzed (16). All the parameters were set to the default value, and the cut-off value was median $=50 \%$. The GBM sample was selected as the dataset and the hazard ratio (HR) was calculated based on the Cox Proportional-Hazards Model. The $95 \%$ CI was not calculated in the present study. For the HR, $\mathrm{P}<0.05$ was considered to indicate a statistically significant difference.

\section{Results}

Screening of DEGs. For the GSE13276 dataset, 429 DEGs were identified, including 279 upregulated (UP) genes and 
Table I. Details of the GEO datasets.

\begin{tabular}{|c|c|c|c|c|c|c|c|c|}
\hline Author, year & Samples & GEO accession & Country & Platform & PBZ, n & $\mathrm{TC}, \mathrm{n}$ & Total, $\mathrm{n}$ & (Refs.) \\
\hline Mangiola et al, 2013 & $\begin{array}{l}\text { GBM WHO grade } \\
\text { IV tumor tissue }\end{array}$ & GSE13276 & Italy & GLP96 & 5 & 5 & 10 & (2) \\
\hline Kruthika et al, 2019 & $\begin{array}{l}\text { GBM WHO grade } \\
\text { IV tumor tissue }\end{array}$ & GSE116520 & India & GPL10558 & 17 & 17 & 34 & (11) \\
\hline
\end{tabular}

GEO, Gene Expression Omnibus; PBZ, peritumoral brain zone; TC, tumor core; GBM, glioblastoma; WHO, World Health Organization.

Table II. Common differentially expressed genes, including 12 upregulated and 63 downregulated genes, of the GSE13276 and GSE116520 datasets.

A, Common upregulated genes

Number of genes Gene symbols

12

CD163, LOX, ABCC3, NAMPT, HILPDA, IGFBP3, TREM1, C8orf4, VEGFA, TGFBI, PI3, CXCL8

B, Common downregulated genes

\begin{tabular}{ll}
\hline Number of genes & \multicolumn{1}{c}{ Gene symbols } \\
\hline 63 & KLK6, GRM3, RAPGEF5, FA2H, PLLP, CAPN3, PPP1R16B, SH3GL3, RAB40B, PTPRD, \\
GPR37, CLCA4, ASPA, PTGDS, SOX10, MBP, HSPA2, ABCA2, MAP7, MYRF, SEC14L5, \\
MAP6D1, PKP4, SHTN1, CHN2, STXBP6, PIP4K2A, PAQR6, NINJ2, MAG, PLEKHB1, \\
SLCO1A2, KCNK1, TSPAN8, ZNF536, SLCO3A1, PLP1, EFHD1, PEX5L, TF, ENPP2, SLC31A2, \\
LHPP, ALDH1A1, UGT8, CYP2J2, MOG, MAL, CNTN2, MOBP, BCAS1, CNTNAP2, RASGRP3, \\
ADARB2, ADAP1, NIPAL3, SEPT4, APLP1, TMEM144, ST18, MVB12B, DAAM2, BIN1 \\
\hline
\end{tabular}

150 downregulated (DOWN) genes. For the GSE116520 dataset, 204 DEGs were identified, including 32 UP genes and 172 DOWN genes. The bioinformatics and evolutionary genomics webpage tool, Venn, was used to construct the Venn diagram of the two groups of DEGs, and through the Venn diagram, 75 common DEGs were identified (Table II), including 12 common UP and 63 common DOWN genes (Fig. 1).

GO term enrichment analysis. The DAVID online tool was used to analyze the GO terms and KEGG pathways of the DEGs. The GO term enrichment analysis results demonstrated that the UP genes were significantly enriched in 'regulation of angiogenesis' $(\mathrm{P}<0.01$; Fig. 2A). DOWN genes were significantly enriched in 'central nervous system development' ( $\mathrm{P}<0.01$; Fig. 2B).

KEGG pathway analysis. By analyzing the DEGs carefully, the most significantly enriched KEGG pathways were identified (Table III). The UP genes were mainly enriched in 'bladder cancer' and the DOWN genes were mainly enriched in 'endocytosis' (Table III).

PPI network and hub gene analysis. STRING online software was used to analyze the DEGs and then a PPI network was constructed to predict the hub genes. Following analysis of the
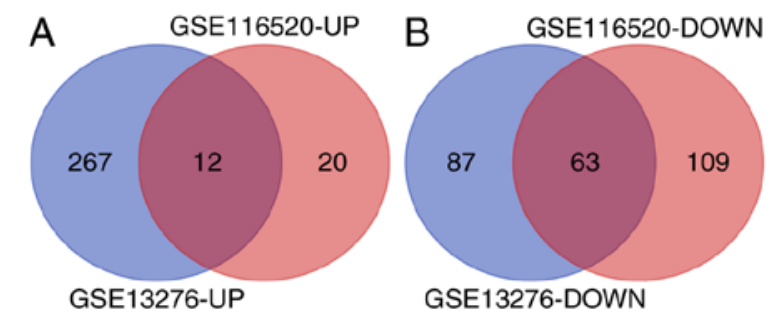

Figure 1. Venn diagrams of common differentially expressed genes in two datasets, constructed using the bioinformatics and evolutionary genomics web tool Venn. (A) Common UP genes in the two datasets. (B) Common DOWN genes in the two datasets. Blue represents the GSE13276 dataset and red represents the GSE116520 dataset. UP, upregulated; DOWN, downregulated.

PPI network, it was identified that 52 nodes and 101 interactions were involved in the PPI network. The top 10 hub genes were calculated using the Degree algorithm of the Cytohubba plug-in, as shown in Fig. 3. The 10 hub genes included proteolipid protein 1 (PLP1), myelin associated oligodendrocyte basic protein (MOBP), contactin 2 (CNTN2), C-X-C motif chemokine ligand 8 (CXCL8), myelin oligodendrocyte glycoprotein (MOG), vascular endothelial growth factor A (VEGFA), myelin basic protein 
Table III. Kyoto Encyclopedia of Genes and Genomes pathway analysis of differentially expressed genes.

\begin{tabular}{llccc}
\hline Term & \multicolumn{1}{c}{ Status } & Count, $\mathrm{n}$ & P-value & \multicolumn{1}{c}{ Genes } \\
\hline hsa05219: Bladder cancer & Upregulated & 2 & 0.023633 & VEGFA, CXCL8 \\
hsa04144: Endocytosis & Downregulated & 4 & 0.049978 & SH3GL3, HSPA2, BIN1, MVB12B
\end{tabular}

VEGFA, vascular endothelial growth factor A; CXCL8, C-X-C motif chemokine ligand 8; SH3GL3, SH3 domain containing GRB2 like 3 , endophilin A3; HSPA2, heat shock protein family A(Hsp70) member 2; BIN1, bridging integrator 1; MVB12B, multivesicular body subunit 12B .
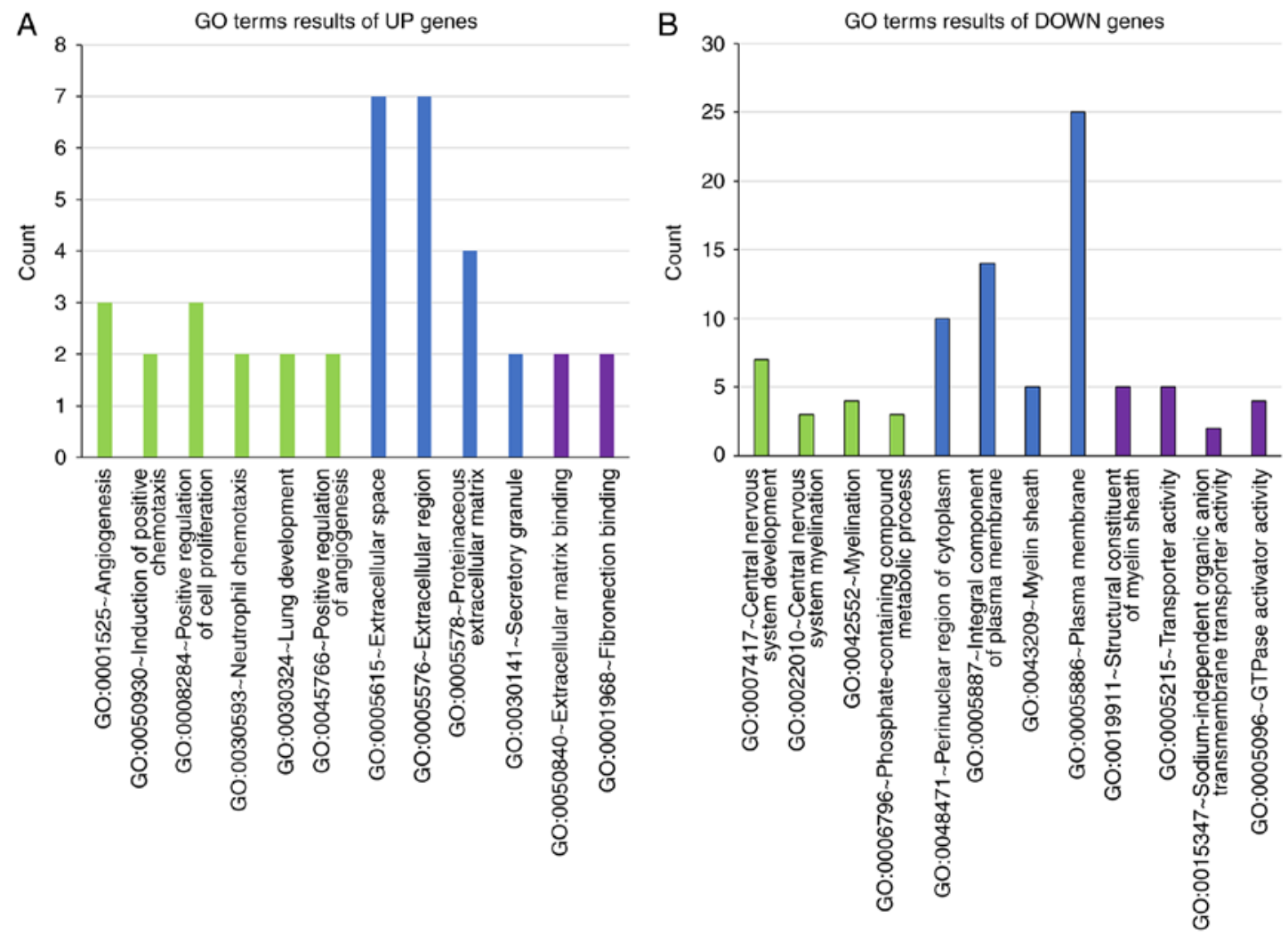

Figure 2. Results of the GO term analysis. (A) GO terms of UP genes. (B) GO terms of DOWN genes. The x-axis represents the enriched GO terms and the $\mathrm{y}$-axis represents the number of genes enriched in the terms. Different colors represent different GO term categories; green represents biological processes, blue represents cellular components and purple represents molecular functions. GO, Gene Ontology; UP, upregulated; DOWN, downregulated.

(MBP), myelin associated glycoprotein (MAG), SRY-box transcription factor 10 (SOX10) and plasmolipin (PLLP). Compared with TC, significant changes in all these hub genes occurred in the PBZ. Additionally, the DAVID database was used for GO enrichment and KEGG pathway analyses of these 10 hub genes. Through GO enrichment analysis, it was determined that the 10 hub genes were mainly enriched in 'cell maturation', 'substantia nigra development' and 'central nervous system development' for biological processes, 'compact myelin' and 'myelin sheath' for cellular components, and 'structural constituent of myelin sheath' for molecular functions (Table IV). According to the KEGG pathway analysis, the 10 genes were enriched in 'bladder cancer' and 'rheumatoid arthritis' (Table V).

Survival analysis. The prognostic value of the 10 hub genes was evaluated using the GEPIA online tool and survival curves were obtained. The GEPIA online tool is based on the TCGA and GTEx databases. Survival analysis of the 10 hub genes revealed that PLP1, MOBP, CNTN2, MOG, MBP, MAG, SOX10 and PLLP were not associated with the overall survival rate of patients with GBM. However, CXCL8 and VEGFA were significantly associated with a reduction in the overall survival rate of patients with GBM (Fig. 4B and D). The expression levels of the 10 hub genes in the normal and tumor groups in the GEPIA database were also compared. The results of the present study demonstrated that the VEGFA and CXCL8 genes expression were differentially expressed in tumor tissues and normal tissues (Fig. 4A and C), and both genes were highly expressed in the PBZ region.

\section{Discussion}

In the present study, to investigate the causes of GBM recurrence, the DEGs between the PBZ and TC were evaluated by bioinformatics analysis. The GSE13276 and GSE116520 datasets were analyzed and 75 common DEGs were identified, 
Table IV. GO enrichment analysis of 10 hub genes.

A, GOTERM_BP_DIRECT

\begin{tabular}{llll}
\hline Term & Count, $\mathrm{n}$ & P-value & Genes \\
\hline GO:0048469 cell maturation & 3 & $1.59 \times 10^{-4}$ & SOX10, PLP1, VEGFA \\
GO:0021762 substantia nigra development & 3 & $2.96 \times 10^{-4}$ & MAG, PLP1, MBP \\
GO:0007417 central nervous system development & 3 & 0.001764 & CNTN2, MOG, MBP \\
GO:0008366 axon ensheathment & 2 & 0.003746 & PLP1, MBP \\
GO:0022010 central nervous system myelination & 2 & 0.003746 & PLP1, CNTN2 \\
GO:0050930 induction of positive chemotaxis & 2 & 0.008013 & VEGFA, CXCL8 \\
GO:0002052 positive regulation of neuroblast proliferation & 2 & 0.010671 & SOX10, VEGFA \\
GO:0031623 receptor internalization & 2 & 0.022817 & CNTN2, CXCL8 \\
GO:0007155 cell adhesion & 3 & 0.023633 & MAG, CNTN2, MOG \\
GO:0042552 myelination & 2 & 0.024392 & PLLP, MBP \\
\hline
\end{tabular}

\section{B, GOTERM_CC_DIRECT}

\begin{tabular}{lccc}
\hline Term & Count, $n$ & P-value & Genes \\
\hline GO:0043218 compact myelin & 2 & 0.001974 & PLLP, MBP \\
GO:0043209 myelin sheath & 3 & 0.002394 & PLP1, MOBP, CNTN2 \\
\hline
\end{tabular}

\section{C, GOTERM_MF_DIRECT}

\begin{tabular}{lccc}
\hline Term & Count, $n$ & P-value & Genes \\
\hline GO:0019911 structural constituent of myelin sheath & 4 & $1.26 \times 10^{-8}$ & PLP1, MOBP, PLLP, MBP
\end{tabular}

GO, Gene Ontology; BP, biological process; CC, cellular component; MF, molecular function; PLP1, proteolipid protein 1; MOBP, myelin associated oligodendrocyte basic protein; CNTN2, contactin 2; CXCL8, C-X-C motif chemokine ligand 8; MOG, myelin oligodendrocyte glycoprotein; VEGFA, vascular endothelial growth factor A; MBP, myelin basic protein; MAG, myelin associated glycoprotein; SOX10, SRY-box transcription factor 10; PLLP, plasmolipin.

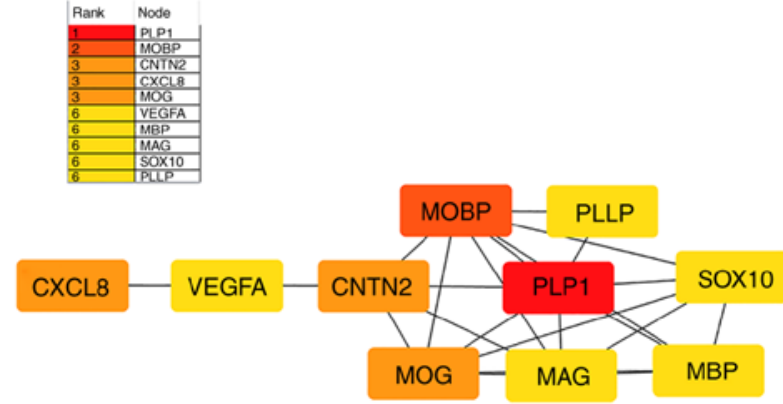

Figure 3. Top 10 hub genes obtained from the analysis of the protein-protein interaction networks, constructed using Cytohubba, a plug-in of Cytoscape. Different colors represent the different importance of the hub genes, in terms of their degree of connectivity. The redder the color block is, the more important it is to other genes. PLP1, proteolipid protein 1; MOBP, myelin associated oligodendrocyte basic protein; CNTN2, contactin 2; CXCL8, C-X-C motif chemokine ligand 8; MOG, myelin oligodendrocyte glycoprotein; VEGFA, vascular endothelial growth factor A; MBP, myelin basic protein; MAG, myelin associated glycoprotein; SOX10, SRY-box transcription factor 10; PLLP, plasmolipin.

including 12 common UP genes and 63 common DOWN genes. DAVID was used to perform a functional annotation analysis of the 75 DEGs. In the GO analysis, the UP genes were enriched in 'regulation of angiogenesis'. The DOWN genes were enriched in 'central nervous system development'. In the KEGG pathway analysis, UP genes were enriched in 'bladder cancer' and DOWN genes were enriched in 'endocytosis'. Based on this, Cytohubba, a plug-in of Cytoscape, was used to analyze the DEGs, and a total of 10 hub genes were identified, including PLP1, MOBP, CNTN2, MOG, MBP, MAG, SOX10, CXCL8, VEGFA and PLLP. Only CXCL8 and VEGFA were UP genes, and the other hub genes were DOWN genes. In addition, GEPIA was used to assess the prognostic value of the 10 hub genes. It was determined that the PLP1, MOBP, CNTN2, MOG, MBP, MAG, SOX10 and PLLP genes did not reduce the overall survival rate of patients with GBM. However, it was demonstrated that CXCL8 and VEGFA were significantly associated with a reduction in the overall survival rate of patients with GBM.

GO term analysis of the 10 hub genes demonstrated that these genes were significantly enriched in terms such as 'cell maturation', 'substantia nigra development' and 'central nervous system development'. In particular, three key genes, CNTN2, MOG and MBP, were enriched in the 'central nervous 
Table V. Kyoto Encyclopedia of Genes and Genomes pathway analysis of 10 hub genes.

\begin{tabular}{lccr}
\hline Term & Count, $n$ & P-value & Genes \\
\hline hsa05219: Bladder cancer & 2 & 0.017777 & VEGFA, CXCL8 \\
hsa05323: Rheumatoid arthritis & 2 & 0.037894 & VEGFA, CXCL8
\end{tabular}

CXCL8, C-X-C motif chemokine ligand 8; VEGFA, vascular endothelial growth factor A.
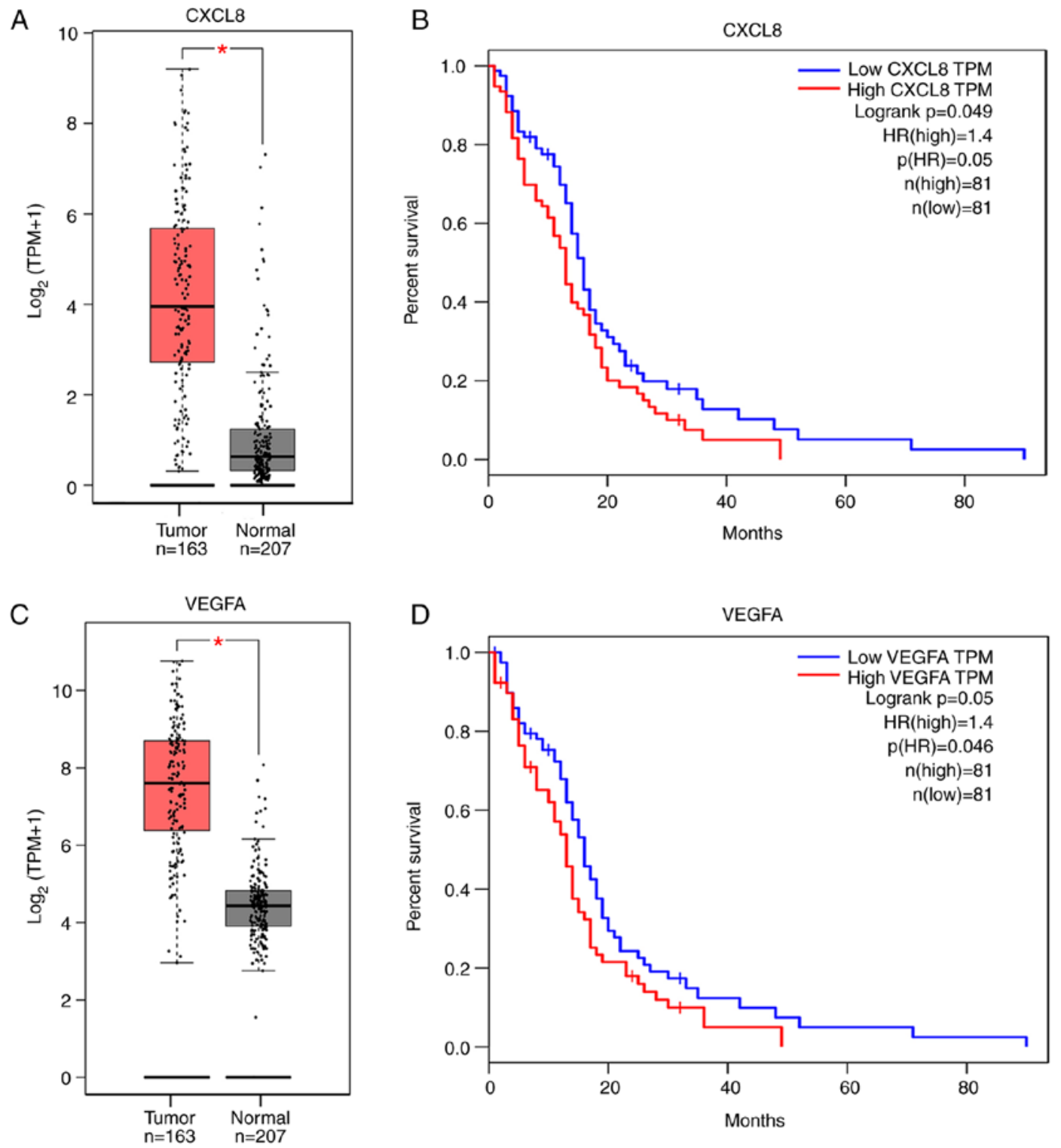

Figure 4. Association between hub genes and overall survival rate in patients with glioblastoma. For the HR value, a "P-value $<0.05$ was considered to indicate an association with overall survival. Expression levels of (A) CXCL8 and (C) VEGFA genes in the tumor (red) and normal (gray) groups. The x-axis represents the groups and the y-axis represents the expression level of the gene. Survival analysis of (B) CXCL8 and (D) VEGFA genes. Red lines represent high expression and blue lines represent low expression of the hub genes. CXCL8, C-X-C motif chemokine ligand 8; VEGFA, vascular endothelial growth factor A; HR, hazard ratio; TPM, transcripts per million.

system development' term. Rickman et al (17) reported that CNTN2 gene expression is increased in middle and high-grade gliomas. Abnormal CNTN2 gene expression is associated with glioma (17). Inhibiting the expression of CNTN2 can inhibit the migration of glioma $(17,18)$. Using the UniProt website (https://www.uniprot.org/), it has been demonstrated that MOG gene expression was associated with cell adhesion (19). In 1994, Nakagawa et al (20) measured the expression level of myelin basic protein (MBP) in the cerebrospinal fluid of patients with various types of tumors, including glioblastoma, 
using radioimmunoassay. It was reported that the expression level of MBP was $>4.0 \mathrm{ng} / \mathrm{ml}$, which was associated with active malignant tumors. In the pathways of 'cell maturation' and 'substantia nigra development', it was indicated that the SOX10, PLP1, and MAG genes were associated with GBM. In 2011, Serão et al (21) found that the SOX10 gene was a high-risk factor for GBM in different races, and Kong et al (22) found that the expression of the PLP1 gene was associated with the morphological classification of GBM. Ljubimova et al (23) found that the MAG gene cannot be expressed in normal brain tissue, but its expression level in GBM was high. In this study by analyzing these genes in 'cell maturation', 'substantia nigra development' and 'central nervous system development' pathways, it was determined that these genes are associated with GBM recurrence.

In the KEGG pathway analysis, the 10 hub genes were enriched in 'bladder cancer' and 'rheumatoid arthritis'. Both pathways involved the two genes VEGFA and CXCL8. The two genes are involved in the angiogenesis of the pathway and promote the progression of these two diseases $(24,25)$. A previous study demonstrated that the use of an anti-rheumatoid arthritis drug (auranofin) inhibits the invasiveness of GBM cells (26). Based on the consideration of these pathways, Lanzardo et al (27) revealed that $\alpha \mathrm{V} \beta 3$ is a target marker of rheumatoid arthritis. Additionally, it has been demonstrated that $\alpha \mathrm{V} \beta 3$ could be used as a marker of GBM molecular imaging (27). The enrichment of these two pathways suggested that the PBZ is more susceptible to the expression of these two genes, which leads to the formation of blood vessels and the possibility of GBM metastasis.

The VEGFA gene can encode angiogenic factors, and its products can alter the microenvironment of endothelial cells and promote angiogenesis (28). In numerous tumors, gene expression products of VEGFA not only stimulate tumor growth, metastasis and survival, but also stimulate hemopoietic cells, endothelial cells and neuronal cells (29-31). Desbaillets et al (32) reported that hypoxia stimulates VEGFA expression in tumor cells in the pseudopalisades region. Wong et al (33) demonstrated that GBM is a type of tumor that is rich in blood vessels and that VEGFA is upregulated in cancer, providing insight to develop anti-VEGF and other anti-angiogenic medicines. Gong et al (34) found that the growth and invasiveness of GBM are associated with the regulation of matrix metalloproteinase 2 (MMP2) by VEGFA. It has also been suggested that blocking MMP2 expression could inhibit angiogenesis when the anti-VGEFA gene is expressed (34). Egorova et al (35) have demonstrated that small interfering RNA can be used to target and knockout VEGFA gene expression in tumor and endothelial cells. Bevacizumab (an antibody that specifically binds to VEGFA) has been found to reduce vascular density and heterogeneity of tumors, but also increases the invasiveness of tumors and causes cognitive impairment in patients $(36,37)$.

The CXCL8 gene has a number of aliases, one of which is interleukin (IL)-8, and is a chemokine with 77 amino acids. Previous studies have demonstrated that it can promote the formation of blood vessels (38-41). Furthermore, Desbaillets et al (32) identified IL-8 gene expression in necrotic cells surrounding GBM, and demonstrated that hypoxia and anoxia could promote IL-8 gene expression.
Additionally, the deficiency of glucose and amino acids, and the increase of reactive oxygen species could promote IL-8 expression (42). Ahn et al (43) reported that necrotic cells induce NF- $\kappa \mathrm{B}$ /activator protein-1, which could promote IL-8 expression in tumor cells. Previous studies have demonstrated that IL- 8 promotes the growth and metastasis of GBM by stimulating its receptors $\mathrm{C}-\mathrm{X}-\mathrm{C}$ chemokine receptor type (CXCR)1 and CXCR2 (44-46). A recent study has demonstrated that bradykinin can induce IL-8 expression through the $\mathrm{B} 1$ bradykinin receptor $(\mathrm{B} 1 \mathrm{R})$, whereas $\mathrm{B} 1 \mathrm{R}$ promotes the transfer of STAT3 and transcription factor SP-1 to the nucleus, and promotes the metastasis of GBM cells (47). Hasan et al (48) analyzed 17 matched pairs of primary and rGBM samples; IL-8 expression was increased in 11 pairs of rGBM samples. Furthermore, blocking IL- 8 gene expression may reduce the expression of key glioma-initiating cells genes, temozolomide (TMZ)-induced increases in transcription factors (Sox2 and C-myc), and enhance the efficacy of TMZ therapy to prolong survival time (48). In accordance with Desbaillets et al (32), this study also found that the IL-8 gene is highly expressed in the PBZ, which may be a signal of migration and proliferation for tumor cells. By targeting CXCR2 using the inhibitor SB225002 (49), knockout of cyclophilin A can reduce the activity of $N F-\kappa B$, inhibit the expression of IL-8, and prevent the metastasis and growth of tumors (50).

CXCL8 and VEGFA genes serve an important role in the growth, proliferation and metastasis of tumors in GBM (29,38-41). However, the principle of using anti-VEGF agents to reduce tumor perfusion, and cause ischemia and hypoxia (51), may additionally promote the expression of vascular endothelial growth factor and IL-8 following hypoxia, and in turn stimulate the invasiveness of tumors. At present, bevacizumab is mainly used in the treatment of rGBM; however, it is not able to prolong overall survival (36), and the recurrence of GBM mainly depends on the surgery (52). Combined with the results of the present study, it may be suggested that both anti-VEGF and anti-IL-8 therapy may be used to improve the prognosis of patients. At present, it was determined that both the VEGFA and CXCL8 genes are highly expressed in the PBZ of GBM, which can cause peripheral angiogenesis, migration and proliferation of tumor cells (29,38-41). Additionally, it was determined that through data analysis, the high expression levels of both VEGFA and CXCL8 can reduce the overall survival rate of patients. Taking into consideration the results of Desbaillets et al (32) and this study, it is suggested that high expression of CXCL8 and VEGFA genes were associated with the recurrence of GBM. However, the association of GBM with recurrence requires further investigation. The gene-related pathways have become increasingly clear. However, further studies are required to understand these pathways and to develop appropriate drugs to improve the prognosis of patients.

In conclusion, through a comprehensive bioinformatics analysis, 10 hub DEGs between PBZ and TC were identified, including PLP1, MOBP, CNTN2, MOG, MBP, MAG, SOX10, VEGFA, CXCL8 and PLLP. The GO and KEGG enrichment analyses revealed that the hub genes were mainly enriched in 'central nervous system development' and 'rheumatoid 
arthritis'. According to literature, the genes in these pathways have a specific association with tumor invasion, metastasis and angiogenesis $(24,25)$, and pathway and term analyses can provide further insights for the development of future drugs and treatments. Through survival analysis of the 10 hub genes, it was demonstrated that high expression levels of the VEGFA and CXCL8 genes are associated with a reduced survival time of patients with GBM, and they were expressed differently in the tumor and normal samples. The present study could provide targets for future therapies and novel insights to block gene expression, which may reduce the recurrence rate of tumors.

\section{Acknowledgements}

Not applicable.

\section{Funding}

No funding was received.

\section{Availability of data and materials}

The datasets generated and/or analyzed during the current study are available in the GEO repository (https://www.ncbi.nlm. nih.gov/geo/query/acc.cgi?acc=GSE13276) and (https://www. ncbi.nlm.nih.gov/geo/query/acc.cgi?acc=GSE116520).

\section{Authors' contributions}

XLu, SX and FY designed the study. XLu, SX, YZ, TT, YX, $\mathrm{XLi}$ and BW performed the bioinformatics analysis and interpretation of the data. XLu, SX and TT wrote the manuscript. FY revised the manuscript and gave final approval of the version to be published. All authors read and approved the final manuscript.

\section{Ethics approval and consent to participate}

Not applicable.

\section{Patient consent for publication}

Not applicable.

\section{Competing interests}

The authors declare that they have no competing interests.

\section{References}

1. Louis DN, Perry A, Reifenberger G, von Deimling A, Figarella-Branger D, Cavenee WK, Ohgaki H, Wiestler OD, Kleihues P and Ellison DW: The 2016 World Health Organization Classification of tumors of the central nervous system: A summary. Acta Neuropathol 131: 803-820, 2016.

2. Mangiola A, Saulnier N, De Bonis P, Orteschi D, Sica G, Lama G, Pettorini BL, Sabatino G, Zollino M, Lauriola L, et al: Gene expression profile of glioblastoma peritumoral tissue: An ex vivo study. PLoS One 8: e57145, 2013.

3. Jayamanne D, Wheeler H, Cook R, Teo C, Brazier D, Schembri G, Kastelan M, Guo L and Back MF: Survival improvements with adjuvant therapy in patients with glioblastoma. ANZ J Surg 88: 196-201, 2018.
4. Lassman AB, van den Bent MJ, Gan HK, Reardon DA, Kumthekar P, Butowski N, Lwin Z, Mikkelsen T, Nabors LB, Papadopoulos KP, et al: Safety and efficacy of depatuxizumab mafodotin + temozolomide in patients with EGFR-amplified, recurrent glioblastoma: Results from an international phase I multicenter trial. Neuro Oncol 21: 106-114, 2019.

5. Franceschi E, Minichillo S and Brandes AA: Pharmacotherapy of glioblastoma: Established treatments and emerging concepts. CNS Drugs 31: 675-684, 2017.

6. Mangiola A, de Bonis P, Maira G, Balducci M, Sica G, Lama G, Lauriola L and Anile C: Invasive tumor cells and prognosis in a selected population of patients with glioblastoma multiforme. Cancer 113: 841-846, 2008.

7. Long H, Liang C, Zhang X, Fang L, Wang G, Qi S, Huo H and Song Y: Prediction and analysis of key genes in glioblastoma based on bioinformatics. Biomed Res Int 2017: 7653101, 2017.

8. Ruiz-Ontañon P, Orgaz JL, Aldaz B, Elosegui-Artola A, Martino J, Berciano MT, Montero JA, Grande L, Nogueira L, Diaz-Moralli S, et al: Cellular plasticity confers migratory and invasive advantages to a population of glioblastoma-initiating cells that infiltrate peritumoral tissue. Stem Cells 31: 1075-1085, 2013.

9. Van Meter T, Dumur C, Hafez N, Garrett C, Fillmore H and Broaddus WC: Microarray analysis of MRI-defined tissue samples in glioblastoma reveals differences in regional expression of therapeutic targets. Diagn Mol Pathol 15: 195-205, 2006.

10. Lama G, Mangiola A, Anile C, Sabatino G, De Bonis P, Lauriola L, Giannitelli C,La Torre G, Jhanwar-Uniyal M, Sica G and Maira G: Activated ERK1/2 expression in glioblastoma multiforme and in peritumor tissue. Int J Oncol 30: 1333-1342, 2007.

11. Kruthika BS, Jain R, Arivazhagan A, Bharath RD, Yasha TC, Kondaiah P and Santosh V: Transcriptome profiling reveals PDZ binding kinase as a novel biomarker in peritumoral brain zone of glioblastoma. J Neurooncol 141: 315-325, 2019.

12. Kanehisa M and Goto S: KEGG: Kyoto encyclopedia of genes and genomes. Nucleic Acids Res 28: 27-30, 2000.

13. Huang DW, Sherman BT and Lempicki RA: Systematic and integrative analysis of large gene lists using DAVID bioinformatics resources. Nat Protoc 4: 44-57, 2009.

14. Huang DW, Sherman BT and Lempicki RA: Bioinformatics enrichment tools: Paths toward the comprehensive functional analysis of large gene lists. Nucleic Acids Res 37: 1-13, 2009.

15. Szklarczyk D, Gable AL,LyonD, Junge A, WyderS, Huerta-Cepas J, Simonovic M, Doncheva NT, Morris JH, Bork P, et al: STRING v11: Protein-protein association networks with increased coverage, supporting functional discovery in genome-wide experimental datasets. Nucleic Acids Res 47: D607-D613, 2019.

16. Tang Z, Li C, Kang B, Gao G, Li C and Zhang Z: GEPIA: A web server for cancer and normal gene expression profiling and interactive analyses. Nucleic Acids Res 45: W98-W102, 2017.

17. Rickman DS, Tyagi R, Zhu XX, Bobek MP, Song S, Blaivas M, Misek DE, Israel MA, Kurnit DM, Ross DA, et al: The gene for the axonal cell adhesion molecule TAX-1 is amplified and aberrantly expressed in malignant gliomas. Cancer Res 61: 2162-2168, 2001

18. Eckerich C, Zapf S, Ulbricht U, Müller S, Fillbrandt R, Westphal $\mathrm{M}$ and Lamszus $\mathrm{K}$ : Contactin is expressed in human astrocytic gliomas and mediates repulsive effects. Glia 53: 1-12, 2006.

19. Solly SK, Daubas P, Monge M, Dautigny A and Zalc B: Functional analysis of the mouse myelin/oligodendrocyte glycoprotein gene promoter in the oligodendroglial CG4 cell line. J Neurochem 68: 1705-1711, 1997.

20. Nakagawa $H$, Yamada $M$, Kanayama $T$, Tsuruzono $K$, Miyawaki Y, Tokiyoshi K, Hagiwara Y and Hayakawa T: Myelin basic protein in the cerebrospinal fluid of patients with brain tumors. Neurosurgery 34: 825-833, 1994.

21. Serão NV, Delfino KR, Southey BR, Beever JE and Rodriguez-Zas SL: Cell cycle and aging, morphogenesis, and response to stimuli genes are individualized biomarkers of glioblastoma progression and survival. BMC Med Genomics 4: 49, 2011.

22. Kong J, Cooper LA, Wang F, Gao J, Teodoro G, Scarpace L, Mikkelsen T, Schniederjan MJ, Moreno CS, Saltz JH and Brat DJ: Machine-based morphologic analysis of glioblastoma using whole-slide pathology images uncovers clinically relevant molecular correlates. PLoS One 8: e81049, 2013.

23. Ljubimova JY, Wilson SE, Petrovic LM, Ehrenman K, Ljubimov AV, Demetriou AA, Geller SA and Black KL: Novel human malignancy-associated gene (MAG) expressed in various tumors and in some tumor preexisting conditions. Cancer Res 58: 4475-4479, 1998. 
24. Reis ST, Leite KR, Piovesan LF, Pontes-Junior J, Viana NI, Abe DK, Crippa A, Moura CM, Adonias SP, Srougi M and Dall'Oglio MF: Increased expression of MMP-9 and IL-8 are correlated with poor prognosis of Bladder Cancer. BMC Urol 12: $18,2012$.

25. Pathak JL, Bakker AD, Verschueren P, Lems WF, Luyten FP, Klein-Nulend J and Bravenboer N: CXCL8 and CCL20 enhance osteoclastogenesis via modulation of cytokine production by human primary osteoblasts. PLoS One 10: e0131041. 2015.

26. Kast RE: Glioblastoma invasion, cathepsin B, and the potential for both to be inhibited by auranofin, an old anti-rheumatoid arthritis drug. Cent Eur Neurosurg 71: 139-142, 2010.

27. Lanzardo S, Conti L, Brioschi C, Bartolomeo MP, Arosio D, Belvisi L, Manzoni L, Maiocchi A, Maisano F and Forni G: A new optical imaging probe targeting $\alpha \mathrm{V} \beta 3$ integrin in glioblastoma xenografts. Contrast Media Mol Imaging 6: 449-458, 2011

28. Bergers $G$ and Benjamin LE: Tumorigenesis and the angiogenic switch. Nat Rev Cancer 3: 401-410, 2003.

29. Wu Y, Hooper AT, Zhong Z, Witte L, Bohlen P, Rafii S and Hicklin DJ: The vascular endothelial growth factor receptor (VEGFR-1) supports growth and survival of human breast carcinoma. Int J Cancer 119: 1519-1529, 2006.

30. Kane NM, Xiao Q, Baker AH, Luo Z, Xu Q and Emanueli C: Pluripotent stem cell differentiation into vascular cells: A nove technology with promises for vascular re(generation). Pharmacol Ther 129: 29-49, 2011.

31. Calvo CF, Fontaine RH, Soueid J, Tammela T, Makinen T, Alfaro-Cervello C, Bonnaud F, Miguez A, Benhaim L, $\mathrm{Xu}$ Y, et al: Vascular endothelial growth factor receptor 3 directly regulates murine neurogenesis. Genes Dev 25: 831-844, 2011.

32. Desbaillets I, Diserens AC, Tribolet N, Hamou MF and Van Meir EG: Upregulation of interleukin 8 by oxygen-deprived cells in glioblastoma suggests a role in leukocyte activation, chemotaxis, and angiogenesis. J Exp Med 186: 1201-1212, 1997.

33. Wong ML, Prawira A, Kaye AH and Hovens CM: Tumour angiogenesis: Its mechanism and therapeutic implications in malignant gliomas. J Clin Neurosci 16: 1119-1130, 2009.

34. Gong J, Zhu S, Zhang Y and Wang J: Interplay of VEGFa and MMP2 regulates invasion of glioblastoma. Tumour Biol 35 : 11879-11885, 2014.

35. Egorova AA, Maretina MA and Kiselev AV: VEGFA gene silencing in CXCR4-expressing cells via siRNA delivery by means of targeted peptide carrier. Methods Mol Biol 1974: 57-68, 2019.

36. Keunen O, Johansson M, Oudin A, Sanzey M, Rahim SA, Fack F, Thorsen F, Taxt T, Bartos M, Jirik R, et al: Anti-VEGF treatment reduces blood supply and increases tumor cell invasion in glioblastoma. Proc Natl Acad Sci USA 108: 3749-3754, 2011

37. Latzer P, Shchyglo O, Hartl T, Matschke V, Schlegel U, Manahan-Vaughan D and Theiss C: Blocking VEGF by bevacizumab compromises electrophysiological and morphological properties of hippocampal neurons. Front Cell Neurosci 13: 113, 2019.

38. Koch A, Polverini P, Kunkel S, Harlow LA, DiPietro LA, Elner VM, Elner SG and Strieter RM: Interleukin-8 as a macrophage-derived mediator of angiogenesis. Science 258: 1798-1801, 1992.
39. Strieter RM, Kunkel SL, Elner VM, Martonyi CL, Koch AE, Polverini PJ and Elner SG: Interleukin-8. A corneal factor that induces neovascularization. Am J Pathol 141: 1279-1284, 1992.

40. Keane MP and Strieter RM: The role of CXC chemokines in the regulation of angiogenesis. Chem Immunol 72: 86-101, 1999.

41. Tada M, Suzuki K, Yamakawa Y, Sawamura Y, Sakuma S, Abe H, van Meir E and de Tribolet N: Human glioblastoma cells produce 77 amino acid interleukin-8 (IL-8(77)). J Neurooncol 16: 25-34, 1993.

42. Brat DJ, Bellail AC and Van Meir EG: The role of interleukin-8 and its receptors in gliomagenesis and tumoral angiogenesis. Neuro Oncol 7: 122-133, 2005.

43. Ahn SH, Park H, Ahn YH, Kim S, Cho MS, Kang JL and Choi YH: Necrotic cells influence migration and invasion of glioblastoma via NF- $\mathrm{kB} / \mathrm{AP}-1$-mediated IL-8 regulation. Sci Rep 6: 24552, 2016.

44. Sharma I, Singh A, Siraj F and Saxena S: IL-8/CXCR1/2 signalling promotes tumor cell proliferation, invasion and vascular mimicry in glioblastoma. J Biomed Sci 25: 62, 2018.

45. Infanger DW, Cho Y, Lopez BS, Mohanan S, Liu SC, Gursel D, Boockvar JA and Fischbach C: Glioblastoma stem cells are regulated by interleukin-8 signaling in a tumoral perivascular niche. Cancer Res 73: 7079-7089, 2013.

46. Dwyer J, Hebda JK, Le Guelte A, Galan-Moya EM, Smith SS, Azzi S, Bidere N and Gavard J: Glioblastoma cell-secreted interleukin-8 induces brain endothelial cell permeability via CXCR2 PLoS One 7: e45562, 2012.

47. Liu YS, Hsu JW, Lin HY, Lai SW, Huang BR, Tsai CF and Lu DY: Bradykinin B1 receptor contributes to interleukin-8 production and glioblastoma migration through interaction of STAT3 and SP-1. Neuropharmacology 144: 143-154, 2019.

48. Hasan T, Caragher SP, Shireman JM, Park CH, Atashi F, Baisiwala S, Lee G, Guo D, Wang JY, Dey M, et al: Interleukin-8/CXCR2 signaling regulates therapy-induced plasticity and enhances tumorigenicity in glioblastoma. Cell Death Dis 10: 292, 2019.

49. Angara K, Borin TF, Rashid MH, Lebedyeva I, Ara R, Lin PC, Iskander A, Bollag RJ, Achyut BR and Arbab AS: CXCR2-expressing tumor cells drive vascular mimicry in antiangiogenic therapy-resistant glioblastoma. Neoplasia 20: 1070-1082, 2018.

50. Sun S, Wang Q, Giang A, Cheng C, Soo C, Wang CY, Liau LM and Chiu R: Knockdown of CypA inhibits interleukin-8 (IL-8) and IL-8-mediated proliferation and tumor growth of glioblastoma cells through down-regulated NF-KB. J Neurooncol 101: $1-14,2011$.

51. Folkman J: Tumor angiogenesis: Therapeutic implications. $\mathrm{N}$ Engl J Med 285: 1182-1186, 1971.

52. Davis ME: Glioblastoma: Overview of disease and treatment. Clin J Oncol Nurs 20 (Suppl 5): S2-S8, 2016.

This work is licensed under a Creative Commons Attribution-NonCommercial-NoDerivatives 4.0 International (CC BY-NC-ND 4.0) License. 